\title{
FutureJournal
}

\section{A Utilização de Ferramentas Prospectivas em Gerenciamento de Risco de Projetos Estratégicos: Análise dos Institutos de Inovação e Tecnologia do Sistema Fiesc}

\author{
Leonardo Costa \\ Federação das Indústrias do Estado de Santa Catarina (FIESC), Brasil \\ lecosta@fiesc.com.br \\ Sidnei Vieira Marinho \\ Universidade do Vale do Itajaí (UNIVALI), Brasil \\ sidnei@univali.br
}

\section{RESUMO}

Os desafios envolvidos no gerenciamento de projetos estratégicos, em um ambiente altamente exigente e de mudanças constantes, impõem uma gestão mais proativa dos riscos envolvidos nos projetos para que estes mantenham posições competitivas no mercado. Na busca por um sistema que permita às organizações serem mais proativas, surge a hipótese de que as ferramentas prospectivas possam ser utilizadas para diminuir as incertezas intrínsecas à gestão de riscos em projetos. Para construir o estado da arte desta pesquisa, foi utilizado o instrumento Knowledge Development ProcessConstrutivist (ProKnow-C). As análises foram realizadas em três institutos de inovação e tecnologia da Federação das Indústrias de Santa Catarina (Fiesc). Os procedimentos metodológicos para a realização do estudo de caso foram baseados na pesquisa documental e em entrevista semiestruturada, permitindo a interpretação dos resultados por meio da correlação das informações obtidas com o estado da arte das publicações sobre o tema pesquisado. Quanto à contribuição para o estado da arte dos temas pesquisados, considera-se a comprovação empírica de que a utilização de ferramentas prospectivas pode ser utilizada de forma conjunta com a gestão de riscos em projetos, no processo de planejamento e gestão estratégica. As conclusões do trabalho indicam que a utilização das ferramentas de prospecção para gerenciar os riscos dos projetos permite, além de melhorar o processo de identificação de riscos, melhorar a proatividade no tratamento dos riscos prospectados por meio do estabelecimento de planos de gestão de riscos, definição de Roadmapping e planejamentos setoriais ou regionais. 


\title{
FutureJournal
}

PALAVRAS-ChAVE: Ferramentas prospectivas. Gestão de riscos em projetos. Institutos de inovação e tecnologia.

\section{The Use of Prospective Tools in Risk Management of Strategic Projects - Analysis of the Institutes of Innovation and Technology of Fiesc System}

\begin{abstract}
The challenges involved in strategic projects managing, in a highly demanding environment and constant changes, impose a more proactive management of the risks involved with the projects, so they can keep more competitive positions in the market. In the search of a system that allows the organizations to be proactive in managing risks and uncertainties of their projects, the hypothesis emerges that the prospective tools can be used to decrease the intrinsic uncertainties project risk management. To build the subject state of the art research, the instrument was Knowledge Development Process-Construtivist (ProKnow-C). The analyses were performed in three Innovation and Technology Institutes of the Industries Federation fromSanta Catarina State (Fiesc). For performing the case study, the methodological procedures were based on the documentary research and semi-structured interviews, allowing the results interpretation, through the information correlation obtained from the state of art publications on the topic searched. About the contribution to the state of art of respondents themes, it is considered theempirical evidence that the use of prospective tools can be used with the project risk management, in the planning process and strategic management. The conclusions of the study indicate that the use of prospective tools to manage risks of the institutes' projects allows, not only improves projects' risk identification, but also improves the risks treatment proactive, through the establishment of risk management plans and setting roadmapping and sectorial or regional planning.
\end{abstract}




\section{FutureJournal}

FUTURE STUDIES RESEARCH JOURNAL: TRENDS AND STRATEGIES
Profuturo: Programa de Estudos do Futuro Editor Científico: James Terence Coulter Wright Avaliação: Double Blind Review, pelo SEER/OJS Revisão: Gramática, normativa e de layout Recebido em: 09/11/2016 Aprovado em: 25/04/2017

KEY-WORDS: Prospective tools. Project risk management. Innovation and technology institutes. 


\section{INTRODUÇÃO}

Gerir projetos estratégicos de grande complexidade no contexto atual, em que mudanças e exigências das partes interessadas são cada vez mais dinâmicas e a competição cada vez mais acirrada, despertou a necessidade de uma avaliação de riscos e de incertezas relacionadas a projetos estratégicos utilizando-se ferramentas prospectivas.

$\mathrm{Na}$ busca por um sistema que permita às organizações serem proativas na gestão dos riscos e das incertezas de seus projetos, surge a hipótese de que as ferramentas prospectivas possam vir a ser utilizadas para estabelecer as possibilidades existentes, ampliando a capacidade de as organizações proporem e monitorarem suas ações estratégicas de forma a permitir, atingir e manter posições competitivas em seus ambientes de negócio, de acordo com determinado ambiente competitivo. Corroborando essa ideia, Raz, Shenhar e Dvir (2002) afirmaram que, em tempos de aumento da concorrência e da globalização, o sucesso do projeto se torna ainda mais crítico para o desempenho dos negócios.

Motawa, Anumba e El-Hamalawi (2006) afirmaram que a proatividade na gestão de projetos é necessária, porém pouco utilizada, pois requer estimativas da probabilidade de ocorrência de eventos, prática pouco presente na gestão de projetos realizada pelas organizações. Já para Koivisto et al. (2009), as ferramentas prospectivas e os processos de gestão dos riscos são utilizados de forma paralela nas organizações, ambos exploram futuros desenvolvimentos plausíveis, avaliam sua conveniência, importância e aceitabilidade, e consideram os riscos envolvidos. Em outras palavras, os autores afirmam que, apesar de utilizarem processos e objetivos semelhantes, as técnicas e ferramentas são utilizadas de forma isolada, o que impede o ganho sinérgico da aplicação conjunta.

Para Raz et al. (2002), mais complicado que a aplicação isolada das técnicas e metodologias para gestão de riscos ou metodologias prospectivas é a não utilização delas, o que geralmente explica o fato de muitos projetos sofrerem atrasos, insatisfações, entregas parciais, custos adicionais e, até mesmo, fracasso. Ironicamente, no entanto, ferramentas e técnicas de gestão de riscos desenvolvidas para melhorar o sucesso da gestão do projeto 
são pouco usadas e muitos ainda se perguntam como elas podem ser úteis. Corroborando essa ideia, para Zeng, An e Smith (2007), pode ser extremamente difícil avaliar os riscos associados a um projeto devido à grande variedade de incertezas envolvida. Amer, Daim e Jetter (2013) ressaltaram que cenários são utilizados para incorporar e enfatizar os aspectos do ambiente no qual estão inseridos e que são importantes para analisar o resultado de projetos.

Para analisar com maior profundidade a utilização integrada de ferramentas prospectivas e metodologias de gestão de riscos foi realizada uma avaliação das atuais práticas de gerenciamento de riscos em projetos e estudos prospectivos, por meio da análise da aplicação empírica no âmbito da Federação das Indústrias de Santa Catarina (Fiesc), mais especificamente em seus projetos estratégicos.

Nos últimos anos, importantes estudos prospectivos têm sido realizados pelas entidades da Confederação Nacional da Indústria (CNI) e Sistema Fiesc e suas entidades (Serviço Social da Indústria - Sesi, Serviço Nacional de Aprendizagem Industrial - Senai, Instituto Euvaldo Lodi - IEL e Centro das Indústrias do Estado de Santa Catarina - Ciesc). Em 2013 foi desenvolvido, pela Unidade de Estudos e Prospectiva (Uniepro) da CNI, juntamente com técnicos do Banco Nacional de Desenvolvimento Econômico e Social (BNDES), um estudo de cenários prospectivos denominado "Cenários integrados: educação profissional e educação básica; inovação e serviços técnicos e tecnológicos; e segurança e saúde no trabalho" (Senai, 2013). Outro importante estudo no âmbito do estado de Santa Catarina e com apoio da Fundação de Amparo a Pesquisa do Estado de Santa Catarina (Fapesc), que está em desenvolvimento, foi denominado de "Setores portadores de futuro para a indústria catarinense - 2022" (Fiesc, 2014) no âmbito do Programa de Desenvolvimento da Indústria Catarinense (PDIC). Esses estudos de cenários foram aplicados no processo de planejamento estratégico vigente, que desde 2013 passou a ser realizado de forma corporativa (Sistema Fiesc).

Esses trabalhos trouxeram valiosas informações sobre o futuro do Sistema Fiesc. Contudo, quando se utilizaram ferramentas prospectivas, um aspecto relevante evidenciado no gerenciamento de riscos dos projetos foi a 
falta de um processo estruturado e sistemático de gestão de riscos ao longo do ciclo de vida dos projetos, de forma a manter a sustentabilidade das soluções criadas por eles.

Com a evolução do processo de gestão estratégica da Fiesc, importantes projetos foram estabelecidos, destacando-se os Institutos de Inovação e Tecnologia do Senai e o Instituto Sesi de Inovação e Tecnologia em Saúde e Segurança do Trabalho, que, por se tratarem de projetos pioneiros e que envolvem valores significativos de investimento, foram escolhidos para ser objeto deste estudo, em que se pretende avaliar a possibilidade de aplicação integrada da gestão de riscos com as ferramentas prospectivas.

Desse modo, a pergunta que emergiu foi: Como é possível utilizar, de forma integrada, as metodologias de gestão de riscos em projetos e ferramentas prospectivas para melhorar a proatividade e a abrangência dos processos de gerenciamento de projetos estratégicos? O objetivo desta pesquisa é, portanto, analisar como as ferramentas prospectivas podem ser utilizadas para o gerenciamento dos riscos em projetos estratégicos dos institutos de inovação e tecnologia do Sistema Fiesc.

Do ponto de vista acadêmico, este trabalho justifica-se, inicialmente, pela sistemática pesquisa bibliográfica, focada em artigos publicados nos periódicos internacionais, indexados em bases disponíveis no portal de periódicos da Capes na última década (2003 a 2014), em que raras publicações tratam de forma integrada os construtos do tema proposto. Adicionalmente, diversos autores reforçaram a importância da pesquisa no campo da gestão de riscos no projeto, como Perminova, Gustafsson e Winkström (2008) quando afirmaram que, apesar de a disciplina de gerenciamento de risco em projeto ter ganho muita atenção da academia na última década, bem como a ampliação de seu uso empírico, ainda há um potencial considerável para o desenvolvimento neste campo.

Para Motawa et al. (2006), a gestão da mudança proativa em projetos é pouco tratada, pois requer estimativas da probabilidade de ocorrência de alterações dos eventos, bem como estimativas do grau de impacto das mudanças sobre parâmetros do projeto. Na mesma linha, Ward e Chapman (2003) ressaltaram que gerenciar um projeto, baseando-se apenas em uma 
perspectiva de ameaça em eventos, pode resultar em uma falta de atenção a várias áreas importantes relacionadas às incertezas do projeto, incluindo a variabilidade decorrente de falta de conhecimento com base em estimativas, o tratamento de suposições sobre as condições de funcionamento, bem como o desenvolvimento de objetivos adequados e compensações associadas aos riscos prospectados.

No âmbito do Sistema Fiesc, o que se espera é melhorar o processo de gestão dos riscos de seus projetos estratégicos, possibilitando a identificação e a atuação proativa sobre os riscos e, por consequência, melhorando os resultados e a sustentabilidade no longo prazo. Espera-se, também, que este novo processo possa orientar melhor a tomada de decisão relacionada a riscos dos projetos estratégicos e inovações de produtos e processos de suas entidades.

Por último, a busca pela aproximação da universidade com a indústria, representa a ligação entre a teoria e a prática, tendo como objetivo mútuo a melhoria da competitividade e da sustentabilidade das organizações e da sociedade como um todo. Para Koivisto et al. (2009), a abertura de um diálogo frutífero entre pesquisadores e profissionais de Future-oriented Technology Analysis (FTA) também facilita a aprendizagem mútua por meio da FTA e das comunidades de avaliação de risco.

\section{FUNDAMENTAÇÃO TEÓRICA}

Nesta seção, será apresentada a teoria relevante sobre a aplicação conjunta das metodologias de gestão de riscos em projetos e as metodologias de ferramentas prospectivas, analisando-se importantes estudos relacionados ao tema.

\subsection{INTEGRANDO A GESTÃO DE RISCOS COM FERRAMENTAS PROSPECTIVAS}

Diante de mercados turbulentos, acirramento da concorrência e das crescentes demandas das partes interessadas, a necessidade de adaptar as ferramentas e as metodologias utilizadas pelas organizações para gerenciar 
os riscos relacionados a projetos precisa ser mais proativa, gerando melhores respostas às necessidades de mudança e, também, mais sistêmica em resposta à maior complexidade que os desafios contemporâneos impõem aos projetos. Nesse sentido, a hipótese subjacente a esta pesquisa é a de que o uso de ferramentas prospectivas possa apoiar o processo de gerenciamento de risco em projetos, de forma a melhorar seus processos e a gestão dos riscos, minimizando as incertezas, tornando-os mais proativos, sistêmicos e sustentáveis.

Koivisto et al. (2009) apresentaram a potencialidade de integrar as ferramentas prospectivas (FTA) com as metodologias de gestão de riscos, objetivando desenvolver avaliação risco mais proativa e também incluir a avaliação sistêmica de risco em tecnologia voltada para a análise do futuro. Para os autores, as características comuns e complementares de ferramentas prospectivas e a gestão de riscos devem ser discutidas, eles sugerem novas formas de evoluir o design modular ao integrar FTA e metodologias de avaliação de risco. Afirmam, ainda, que em resposta ao mundo cada vez mais complexo criam-se outros tipos de riscos que não devem ser ignorados na análise de oportunidades futuras, na criação de visões compartilhadas e na avaliação dos impactos desejados.

Também na linha do uso integrado desses construtos, Barros, Werner e Travassos (2004) apresentaram uma abordagem para desenvolver, recuperar e reutilizar a gestão do conhecimento humano e suas experiências, preocupados com os riscos no desenvolvimento de projetos de software. Esses autores demonstram a utilização de cenários para modelar o impacto de riscos e estratégias de resolução de eficácia dentro de arquétipos de risco. O processo proposto pelos autores assemelha-se a uma estrutura de processo de reaproveitamento, em que dois subprocessos são, respectivamente, responsáveis por identificar e reutilizar informação de risco. Simular o impacto dos riscos esperados pode suportar algumas das decisões de todo o projeto de desenvolvimento do software. A contribuição deste trabalho é mostrar como arquétipos de risco e as ferramentas prospectivas podem representar o reaproveitamento dos conhecimentos para o gerenciamento de projeto. 
Nessa mesma linha de pensamento, Koivisto et al. (2009) afirmaram que a criação de uma base de conhecimento mais ampla e o aprendizado por meio dessas disciplinas são considerados importantes para uma gestão de riscos mais proativa e sistêmica, que abranja até mesmo novos tipos de riscos emergentes, incluindo aqueles relacionados às novas tecnologias e a sua introdução no mercado. Por outro lado, espera-se que o desenvolvimento de metodologias de avaliação prospectiva também se beneficie das experiências da tradição de avaliação de risco.

Em geral, as ferramentas prospectivas incentivam a construção de novas técnicas de análise de risco, permitindo que estas sejam mais capazes de levar em consideração o maior tempo necessário para maturação dos projetos do que os métodos tradicionais. Adicionalmente, a gestão de riscos é beneficiada pelas ferramentas de prospectiva, tornando os pontos de vista mais holísticos. Para Barros et al. (2004), o paradigma de gerenciamento de projetos baseado em cenários compreende um conjunto de técnicas que permite que um gerente possa definir o comportamento esperado de um projeto em vários diferentes cenários que possam ocorrer ao longo de seu desenvolvimento. Combinações de tais cenários são aplicadas sobre o projeto e usadas para avaliar seu impacto sobre seu comportamento.

Segundo Perminova et al. (2008), muitos estudiosos do gerenciamento de projetos apontam que há uma lacuna entre as tradicionais ferramentas de gerenciamento de risco do projeto, como análise, monitoramento e controle de risco, e aquelas que visam compreender o futuro e seus reflexos. Apesar de os processos de gerenciamento de riscos serem frequentemente vistos como um processo de ciclo de vida, sua aplicação prática mostra que os procedimentos relacionados com a previsão do futuro não se repetem em todas as fases do projeto.

Conforme Motawa et al. (2006), a gestão da mudança proativa em projetos é pouco tratada, pois requer estimativas da probabilidade de ocorrência de alterações dos eventos, bem como estimativas do grau de impacto das mudanças sobre parâmetros do projeto. Nesse sentido, os autores propuseram um sistema fuzzy para manter esses requisitos, pois esse sistema simula as relações entre causas e efeitos das mudanças e destina-se a facilitar a gestão proativa da mudança em projetos. 
Em alinhamento com Motawa et al. (2006), Lopez e Salmeron (2014) apresentaram estudo em que construíram Mapas Cognitivos Fuzzy, destacando que a principal vantagem de Fuzzy Cognitive Maps (FCM) reside em serem capazes de modelar fenômenos complexos com base em percepções dos peritos. Esse modelo de ferramenta relacionado a eventos de incerteza procuram imitar o raciocínio humano. A ferramenta proposta por esses autores especifica as percepções, os riscos e os resultados do projeto de manutenção de Enterprise Resource Planning (ERP), bem como suas interações mais obscuras. Vale destacar que FCM permite o desenvolvimento de exercícios de previsão por meio de simulações e, portanto, a ferramenta proposta ajudaria a gerenciar riscos de projetos de manutenção de ERP de forma mais eficaz e proativa.

Para Motawa et al. (2006), a qualidade da aproximação fuzzy depende predominantemente da qualidade do julgamento da engenharia (conhecimento subjetivo) e da experiência humana usada para identificar as variáveis fuzzy de entrada. Por essa razão, o desenvolvimento de cenários fuzzy para as variáveis de entrada identificadas é muitas vezes considerado o passo mais importante no desenvolvimento de um sistema fuzzy.

A técnica FCM descreve um modelo de mapa cognitivo com duas características significativas: primeira - as relações causais entre nós têm diferentes intensidades, representadas por números fuzzy; e segunda - o sistema é dinâmico. Trata-se de feedback, em que o efeito da mudança de um nó conceito pode afetar outros nós, que por sua vez podem afetar o nó de início da mudança (Lopes \& Salmeron, 2014). Dado um vetor em estado inicial, representando os valores iniciais (no instante zero) de nós de modelo, um FCM pode simular sua evolução ao longo do tempo (no instante $t$ ). Assim, essa técnica pode prever o comportamento futuro do domínio específico representado. O estado das variáveis evolui, dependendo dos valores de peso fuzzy atribuídos aos links de feedback entre as variáveis. Portanto, FCM permite a análise da evolução de um cenário em momentos sucessivos e a avaliação de alternativas, mas aplicando-se uma análise complementar. Para Kosko (1986), o FCM proporciona excelente mecanismo para o desenvolvimento de exercícios de previsão. 
Ainda em relação ao FCM, López (2012) afirmou que ele demonstra o comportamento de um sistema em termos de conceitos, cada conceito representa uma entidade, uma variável, ou uma característica do sistema. As variáveis de estado evoluem dependendo dos valores de peso fuzzy atribuídos à realimentação das ligações entre variáveis. Modelos conjuntivos descrevem a dinâmica de comportamento de sistemas complexos no longo prazo, com base na causa e efeito das relações entre nós. Métodos como FCMs, dinâmica de sistemas, redes bayesianas e redes neurais pertencem a esta abordagem de modelagem.

Vale destacar que Motawa et al. (2006) propuseram ajudar a determinar os riscos com base nas informações disponíveis nas fases iniciais de projetos. Fontes de mudança em projetos de construção incluem as incertezas associadas com o conhecimento impreciso e vago de grandes projetos e de informações nas fases iniciais de projetos. Outras fontes de mudança podem também existir, mas não podem ser determinadas nas fases iniciais dos projetos, portanto, não foram consideradas pelos autores na modelagem proposta. A técnica de modelagem utilizada permite que a equipe do projeto rastreie a origem desses riscos, facilitando o desenvolvimento de uma ação planejada e proativa para lidar com eles, antes de a mudança acontecer.

Apesar de focar apenas nas fases iniciais do projeto, o trabalho dos autores difere da visão deste pesquisador que considera que os riscos podem ser identificados, incorporados e revistos ao longo de todo o ciclo de vida do projeto. Porém, Motawa et al. (2006) demonstraram preocupação com a necessidade da proatividade no tratamento dos riscos. O foco desta pesquisa lida com as mudanças proativas/reativas tomadas e a interdependência entre múltiplas causas e efeitos. O sistema de gerenciamento de mudança desenvolvido consiste em um modelo para identificar e prever potenciais mudanças e avaliar seus efeitos, que por sua vez vai ajudar na estimativa do valor em risco para a mudança. A proposta de Motawa et al. (2006) é muito interessante e alinha-se ao eixo principal desta pesquisa, pois os conceitos de prospectiva também são embasados nas fases iniciais dos projetos.

Para López (2012), FCM é uma ferramenta adaptável que pode ser personalizada a fim de se considerarem as especificidades do fenômeno 
representado. Isso também é muito flexível, pois, se a primeira representação dos elementos é incompleta ou incorreta, novos elementos podem ser adicionados ao mapa e os efeitos dos novos parâmetros podem ser rapidamente vistos. Além disso, a técnica de FCM sofre de algumas limitações. O resultado FCM é altamente dependente da fonte de dados. Em verdade, é necessária uma série de especialistas para garantir resultados objetivos e globalmente válidos.

Em uma visão bem mais ampla em relação à participação de especialistas, Koivisto et al. (2009) afirmaram que também é importante, em todos os campos, a criação de entendimentos compartilhados entre as partes interessadas sobre os possíveis desenvolvimentos futuros. Na avaliação de risco, o foco tipicamente está em resultados negativos, enquanto os estudos prospectivos focam na evolução positiva, bem como enfatizam a possibilidade de inovação, o que amplia ainda mais o conceito. Por isso, de alguma forma, a ligação entre essas duas tradições de pesquisa é, em essência, presente, mas na prática as pesquisas, projetos de pesquisa e estudos de caso separaram-se e raramente são ligados.

\section{METODOLOGIA}

Nesta seção, apresenta-se o enquadramento metodológico da pesquisa, que em síntese trata de um estudo de casos por meio de pesquisa documental e de entrevistas semiestruturadas com os gestores e especialistas dos Institutos de Inovação e Tecnologia da Fiesc, com enfoque na análise dos construtos desta pesquisa.

Do ponto de vista de sua natureza, o estudo é classificado como pesquisa aplicada, pois objetiva gerar conhecimentos para aplicação prática e dirigi-los à solução de problemas específicos dos estudos de caso, bem como envolve verdades e interesses específicos da organização investigada (Gil, 1991).

Especificamente no estudo de caso desta pesquisa, serão analisados os processos de gestão de riscos e utilização de ferramentas prospectivas em três projetos estratégicos relacionados à implantação de institutos de inovação e tecnologia da Federação das Indústrias de Santa Catarina (Fiesc), 
dois do Senai/SC e um do Sesi/SC, de maneira que se permita seu conhecimento. A escolha dos três casos deveu-se à importância estratégica desses projetos para a Fiesc, por estarem em diferentes estágios de implantação (dois em fase de projeto e um já em operação) e também por estarem sendo realizados em parceria com importantes institutos de pesquisa em inovação e tecnologia mundiais, as universidades de Harvard e de Stanford e o Instituto Fraunhofer.

Em relação à forma de abordagem do problema, a pesquisa é qualitativa (Creswell, 1994). No que concerne aos objetivos, o estudo pode ser classificado como pesquisa exploratória. Os procedimentos técnicos utilizados na elaboração deste trabalho, conforme descrito por Gil (1991; 1999), são: pesquisa bibliográfica e documental.

A pesquisa bibliográfica foi elaborada a partir de material já publicado, constituído principalmente de livros, artigos de periódicos, relatórios gerenciais e materiais disponibilizados na Internet. Com o objetivo de construir o estado da arte do tema de pesquisa, o pesquisador utilizou o instrumento Knowledge Development Process-Construtivist (ProKnow-C), para realizar o mapeamento do tema.

Quanto à pesquisa documental, é a etapa de análise dos documentos relacionados à organização pesquisada, em especial os documentos relacionados ao tema deste trabalho (gestão de riscos em projetos e ferramentas prospectivas).

Realizadas a seleção e a análise preliminar dos documentos, o pesquisador procedeu à análise dos dados.

Também é importante ressaltar a convivência do pesquisador com os gestores e executores dos projetos, pois participou ativamente da realidade vivida pelos pesquisados, gestores e especialistas dos institutos de inovação e tecnologia da Fiesc, inclusive apoiando na realização de algumas etapas dos processos de planejamento e da aplicação de ferramentas prospectivas. Essa troca de informações e experiências foi essencial para compreensão das rotinas dos projetos dos institutos e análise dos resultados finais da pesquisa.

A partir das pesquisas bibliográficas e documentais, elaborou-se um roteiro básico de entrevista (Apêndice) para completar o processo de investigação empreendido neste estudo de caso. O foco das entrevistas foi 
analisar, sob a ótica dos principais gestores envolvidos com os projetos estratégicos deste estudo de caso (Institutos Sesi e Senai de Inovação e Tecnologia), como está efetivamente implantado o objetivo principal desta pesquisa, ou seja, analisar como é realizada a gestão de riscos e a utilização de ferramentas prospectivas no gerenciamento de projetos estratégicos nos institutos de inovação e tecnologia do Sistema Fiesc.

As entrevistas foram aplicadas a oito dos principais gestores dos projetos e das instituições responsáveis pelos projetos do objeto deste trabalho no período de onze de fevereiro a treze de abril do ano de 2015. Optou-se por um foco limitado de entrevistados, pois um estudo de caso para ter credibilidade não necessariamente deve ter um grande número de entrevistados, mas sim um pequeno número de pessoas-chave (Yin, 2001). Dessa forma, foram entrevistados:

- o diretor superintendente, o assessor de gestão organizacional e o gestor do projeto do Instituto de Inovação e Tecnologia em Saúde e Segurança no Trabalho do Sesi/Fiesc;

- o gerente e o gestor corporativo dos institutos do Senai/Fiesc do projeto do Instituto Senai de Tecnologia em Automação e TIC;

- o diretor do instituto e o gestor corporativo dos institutos Senai/Fiesc do Instituto Senai de Inovação em Sistemas Embarcados;

- adicionalmente foram entrevistados os gestores das áreas responsáveis por ferramentas prospectivas, gestão de riscos e planejamento estratégico da Fiesc.

O instrumento de intervenção para realizar as entrevistas classificase como semiestruturado, pois, de acordo com May (2004), difere do estruturado por ser de caráter aberto, permitindo ao entrevistado responder as perguntas dentro de sua concepção. Quanto à interpretação dos resultados, o modelo utilizado é o padrão combinado, que permite fazer a análise comparando os padrões da base empírica (pesquisa documental e entrevistas) com os padrões previstos (pesquisa bibliográfica) (Yin, 2001). Se os padrões forem assemelhados, os resultados ajudarão a validar o estudo de caso. 


\section{RESULTADOS}

Este tópico destina-se à análise da possibilidade da aplicação conjunta da gestão de riscos em projetos estratégicos, associada a ferramentas prospectivas por meio do estudo de três casos (institutos de inovação e tecnologia da Fiesc), que se configurou como objetivo principal desta pesquisa. Adicionalmente, realizou-se a análise das técnicas e ferramentas utilizadas e suas formas de abordagem.

\subsection{ANÁLISE DA APLICAÇÃO CONJUNTA DA GESTÃO DE RISCOS EM PROJETOS ESTRATÉGICOS UTILIZANDO-SE FERRAMENTAS PROSPECTIVAS}

A percepção dos gestores em relação à possibilidade de aplicação conjunta foi investigada por meio de seis questionamentos feitos diretamente aos gestores da Fiesc e dos projetos estratégicos da Fiesc (entrevista semiestruturada). Tais percepções estão em alinhamento com os referenciais teóricos desta pesquisa, na busca pelo alinhamento do processo documentado pela organização (análise documental), conforme abordagem dos questionamentos apresentados na sequência.

A análise documental não explicita o uso integrado intencional da gestão de riscos e das ferramentas prospectivas para o gerenciamento de riscos nos projetos estratégicos, apesar de ambas estarem inseridas no processo de planejamento estratégico da Fiesc. Nas entrevistas, a análise pôde ser mais profunda, até mesmo porque as questões buscavam ponderar as percepções dos gestores em relação à utilização conjunta dos construtos.

Na primeira questão deste bloco, que busca entender a percepção dos gestores a respeito da possibilidade de utilização conjunta, as respostas favoráveis foram unânimes, ou seja, todos os entrevistados afirmam que a gestão de riscos de projetos estratégicos pode beneficiar-se da utilização de ferramentas prospectivas, pois permite maior proatividade no tratamento dos riscos. Um exemplo é a resposta do analista de Gestão e Inteligência Estratégica: "Sim, pois estar munido de informações que permitam se antecipar em relação a possíveis eventos futuros é muito interessante, ou 
seja, a prospectiva ajuda a identificar os riscos que poderão afetar o projeto ao longo do seu percurso de implantação".

Na segunda questão, que busca a percepção do quanto a gestão dos riscos em projetos leva em conta os estudos prospectivos construídos pelos institutos, encontram-se diferentes percepções dos gestores entrevistados. Dois entrevistados (diretor corporativo e o analista de Gestão e Inteligência Estratégica) afirmam que hoje essas duas práticas são realizadas de forma isolada, ou seja, não percebem a existência da utilização integrada. Os demais entrevistados percebem que, sim, de alguma forma, a gestão de riscos dos projetos se beneficia das prospectivas, pois permite observar oportunidades e ameaças que o projeto enfrentará. Um exemplo é a afirmação do gestor do projeto do Instituto Senai de Inovação e Tecnologia em Automação e TIC: "O PDIC, durante a elaboração da rota setorial (roadmap) do setor de automação e TIC, demonstrou boas oportunidades para soluções inovadoras a serem desenvolvidas pelo Instituto, nos levando a repensar e revisar o projeto".

Na terceira questão, que visa avaliar o quanto a gestão integrada dos riscos e estudos prospectivos auxilia na gestão dos riscos do projeto e busca evidência empírica disso, todos os entrevistados avaliam positivamente, porém quando demandados a exemplificar "Se sim, como isto pode ser observado?", poucos responderam, ou seja, além do gestor do projeto do Instituto Senai de Inovação e Tecnologia em Automação e TIC (que exemplificou na questão anterior), apenas o coordenador corporativo dos projetos dos institutos respondeu: "Como, por exemplo, a oportunidade de verificar a existência de tecnologia obsoleta, tendências tecnológicas e regulamentações".

Na quarta questão, a busca da pesquisa foi: "Como é avaliada a resposta da sociedade ao convite para que seus representantes possam participar destes estudos prospectivos promovidos pelo Sistema Fiesc?". A análise documental evidencia a importância da efetiva participação das diversas partes interessadas no tema em estudo, bem como a participação efetiva de diversos atores relacionados à vocação de cada instituto de inovação e tecnologia da Fiesc. 
Nas entrevistas, com exceção do diretor corporativo, que afirmou que "faltaram algumas pessoas muito importantes nos nossos Painéis de Especialistas", referindo-se à expectativa não concretizada de participação de alguns empresários de indústrias importantes de Santa Catarina, os demais respondentes foram unânimes na percepção de que houve uma participação efetiva das partes interessadas. Por efetividade, considera-se não apenas a participação de elevado número de pessoas, mas também pessoas qualificadas dentro das organizações que representavam. Saritas e Oner (2004) e Saritas e Aylen (2010) enfatizam a utilidade da ajuda das partes interessadas.

Na quinta questão: "Você concorda que a fase da implementação de estudos de cenários é a parte mais complexa da gestão dos riscos em projetos? Se sim, como a Fiesc monitora a implantação de estudos de cenários para mitigar os riscos encontrados na fase de concepção e ao longo da execução do Projeto?", na análise documental demonstrou-se que existem diferentes formas utilizadas para minimizar potenciais efeitos negativos (fraquezas e ameaças) ou potencializar os efeitos positivos (forças e oportunidades) apontados pelos estudos prospectivos. Nesse sentido, conforme já citado pelos gestores durante as entrevistas, destacam-se o PDIC, pois construiu rotas estratégicas setoriais (roadmap), e o Masterplan. Pôde-se constatar nas entrevistas que não foi possível avaliar plenamente a fase de implantação dos estudos prospectivos em relação a dois casos (Instituto Senai de Inovação em Sistemas Embarcados e Instituto Sesi de Inovação e Tecnologia em Saúde e Segurança do Trabalho), pois ambos estavam em estágios iniciais de construção e implantação e também pelo fato de os estudos prospectivos serem muito recentes. Quanto ao Instituto Senai de Inovação e Tecnologia em Automação e TIC, que já se encontra em um estágio avançado de implantação, as ações prospectadas foram controladas por meio de reuniões mensais e do software Channel. Saritas e Oner (2004) afirmaram que, embora exercícios de previsão tenham sido amplamente praticados, seu sucesso nas implementações não tem sido tão grande, porque a aplicação é a fase mais desafiadora de um estudo prospectivo. Essa afirmação foi corroborada por todos os gestores de projeto dos institutos da Fiesc. 
Na sexta e última pergunta deste bloco: "Existe alguma ferramenta que permita projeções quantitativas dos riscos relacionados aos projetos?", a análise documental, mais especificamente no relatório do Channel, apresenta cálculos de projeções do orçamento no término e projeção para a data de término do projeto. Essas estimativas são calculadas para dois cenários, um deles projetando os valores considerando-se que o projeto mantenha o índice de desempenho atual, e o outro de acordo com os cenários prospectados mais prováveis.

Nas entrevistas ficou evidenciado que esses cálculos e projeções são periodicamente gerenciados por meio de reuniões mensais, em que se estabelecem ações corretivas em caso de não cumprimento de orçamento ou prazo.

Apenas dois entrevistados (diretor corporativo e o analista de gestão e inteligência estratégica) afirmaram desconhecer a existência de ferramentas quantitativas para apoiar a gestão dos riscos do projeto. Os demais entrevistados afirmaram que utilizam ferramentas quantitativas apenas para gerenciar riscos financeiros, como, por exemplo, o gestor corporativo dos projetos dos institutos, que citou a ferramenta EVTEC (Estudo de Viabilidade Técnica), que permite a gestão de investimentos, fluxo de caixa, payback, valor presente líquido (VPL) e taxa interna de retorno (TIR).

Neste ponto, nesta análise aponta-se para uma importante lacuna nos processos atuais de Gestão de Riscos em projetos dos institutos da Fiesc, ou seja, a utilização de técnicas quantitativas pode melhorar e dar consistência para as práticas qualitativas. Van Vliet, Kok, Veldkamp (2010) sugeriram o uso da FCM, que utiliza como base metodológica a lógica Fuzzy, para integrar as análises qualitativa e quantitativa, visão compartilhada por Amer et al. (2013), quando afirmaram que cenários baseados em FCM proporcionam os benefícios da abordagem intuitiva de cenário juntamente com a análise quantitativa.

\subsection{ANÁLISE DAS TÉCNICAS E FERRAMENTAS UTILIZADAS}

A análise das técnicas e ferramentas utilizadas para gerenciar riscos e construir cenários prospectivos foi investigada por meio de três 
questionamentos, feitos diretamente aos gestores da Fiesc e dos projetos estratégicos da Fiesc. Esses questionamentos estão embasados nos referenciais teóricos desta pesquisa e na busca do alinhamento do processo documentado pela organização. Na sequência, será abordado cada um dos questionamentos seguindo essa estrutura.

O primeiro questionamento foi: "Quais as principais técnicas e ferramentas utilizadas para gerenciar os riscos dos projetos?". A análise documental demonstrou que as metodologias e ferramentas utilizadas para gerenciar os riscos do projeto estão embasadas no Project Management Body of Knowledge - PMBOK e na norma ABNT NBR ISO 31000 (ABNT, 2009), ambas reconhecidas como referência na gestão de riscos em projetos, corroborando Ferreira, Almeida, Leão e Silva (2013), quando afirmaram que diversas metodologias foram criadas para gerenciar os riscos e as incertezas relacionadas a projetos, duas das quais merecem destaque: a norma ABNT NBR ISO 31000 (ABNT, 2009) e o guia PMI (PMI, 2012).

As entrevistas demonstraram que há uma diversidade de práticas como as relacionadas à área de planejamento e gestão, foram citadas: sistema Channel, relatórios sistemáticos, reuniões mensais, ABNT NBR ISO 31000 (ABNT, 2009), o Guia PMI (PMI, 2012), Committee of Sponsoring Organizations of the Treadway Commission (COSO) e EVTEC. O diretor corporativo fez uma citação interessante quanto ao uso de Impact Matrix Cross-Reference Multiplication Applied to a Classification (Micmac), que permite a integração com a metodologia Delphi, técnica que pode ser usada para obter consenso a respeito dos riscos de um projeto. O método Delphi é baseado no princípio de que as previsões por um grupo estruturado de especialistas são mais precisas se comparadas às provenientes de grupos não estruturados ou individuais. Cabe destacar que a metodologia Delphi também é muito utilizada para a formulação de cenários e, portanto, reforça a ideia central desta pesquisa, que é o uso integrado da gestão de riscos em projetos com as ferramentas prospectivas. Para os gestores dos institutos, houve unanimidade na citação do uso do EVTEC e do sistema Channel. Uma prática citada de forma isolada pelo gestor do Instituto Senai de Inovação em Sistemas Embarcados, que afirmou utilizar o plano de negócio seguindo a metodologia do Project Model Canvas, uma prática interessante para 
identificação dos riscos relacionados aos negócios (soluções) a serem propostas pelos institutos.

Em síntese, vale destacar que, apesar de diversas práticas terem sido citadas, todas estão alinhadas a metodologias e ferramentas observadas na análise documental e no referencial teórico desta pesquisa.

No segundo questionamento: "Quais as principais técnicas e ferramentas utilizadas para elaborar e gerir os estudos prospectivos?", a análise documental demonstrou que as ferramentas prospectivas utilizadas estão embasadas em Popper, (2008), que, além de apresentar um número significativo de ferramentas, analisou a natureza das metodologias e ferramentas prospectivas, afirmando que elas podem variar entre as baseadas em "evidências" ou "criativas" e "expertise" ou "interação" com métodos de trabalho.

As entrevistas demonstraram que há uma diversidade de ferramentas prospectivas utilizadas. Por exemplo, o grupo de entrevistados relacionados à área de Planejamento e Gestão citou: PDIC, cenários estratégicos, roadmap, painel de especialistas, pesquisa desk e scanning, visão mundo/Brasil/Santa Catarina. Já as ferramentas prospectivas mais citadas pelos gestores dos projetos dos institutos foram: PDIC, painel de especialistas, rotas setoriais (roadmap), projeções econômico-financeiras no plano de negócio (EVTEC).

Nesta questão, ficou evidenciado que os entrevistados são unânimes quanto à citação de utilização do PDIC, do painel de especialistas e do roadmap. As demais ferramentas citadas apenas pelos gestores corporativos justificam-se, pois as demais técnicas são aplicadas em outras etapas dos processos do planejamento e gestão estratégica, ou seja, as demais ferramentas prospectivas não estão sendo utilizadas com foco nos projetos dos institutos, mas nos riscos estratégicos da Fiesc.

O terceiro questionamento foi: "A ferramenta painel de especialistas foi utilizada em todos os estudos prospectivos citados na análise da documentação. Você participou de algum painel? Qual?". A análise documental descreve que a prática do painel de especialistas é amplamente utilizada como uma etapa para atualização e construção das prospectivas da Fiesc. Do painel de especialistas, devem participar especialistas temáticos dos 
departamentos regionais do Sesi, Senai, IEL e áreas corporativas e do departamento nacional, bem como a equipe de prospectiva da Unidade de Estudos e Prospectiva (Uniepro). Já para o PDIC, o painel de especialistas é realizado em cada uma das regiões do estado de Santa Catarina, e os especialistas devem analisar os estudos socioeconômicos previamente elaborados, estudos industriais, tendências em P\&D\&I, além de apoiarem a definição dos Setores Portadores de Futuro para cada região.

As entrevistas reforçaram que todos os respondentes da pesquisa participaram ativamente dos painéis de especialistas, que se configurou como prática amplamente utilizada pela Fiesc.

\subsection{FORMAS DE ABORDAGEM}

A análise das formas de abordagem das técnicas e ferramentas utilizadas para gerenciar riscos nos projetos e construção de ferramentas prospectivas foi realizada por meio de dois questionamentos feitos aos gestores da Fiesc e dos projetos dos institutos da Fiesc. Ambos estão embasados nos referenciais teóricos desta pesquisa e na busca pelo alinhamento do processo documentado pela organização. Na sequência será abordado cada um dos questionamentos.

O primeiro e o segundo questionamentos em relação às formas de abordagem foram: "Em relação à forma de abordar os riscos e estudos prospectivos, as técnicas e ferramentas utilizadas são preponderantemente qualitativas ou quantitativas?" e "Como são realizadas as análises quantitativas dos riscos dos projetos?". A análise documental demonstrou que devem ser utilizadas ferramentas quantitativas e qualitativas, tanto para a gestão dos riscos quanto para os estudos prospectivos, conforme evidenciado no Plano de Gerenciamento de Riscos, que descreve: "A escolha do método de análise é influenciada pelos contextos, pelos objetivos e pelos recursos disponíveis, podendo ser aplicadas análises qualitativas ou quantitativas, ou até mesmo ambas, de acordo com a necessidade". Essa prática está alinhada ao PMI (PMI, 2012, p. 336), pois define que os dois processos principais para gerenciamento de riscos são o processo de planejamento (planejar o gerenciamento de riscos; identificar os riscos; 
realizar análise qualitativa; realizar análise quantitativa; e planejar as respostas aos riscos) e o processo de monitoramento (controlar os riscos).

Ainda em relação à análise documental, mais especificamente quanto ao tipo de abordagem das ferramentas prospectivas, foi relatado que "para a Fiesc, construir cenários significa buscar antecipar eventos futuros através da interpretação quantitativa e qualitativa de pontos críticos dos fatores externos, que têm influência sobre os negócios e as organizações".

Nas entrevistas, houve diferentes interpretações. Segundo os respondentes da área de Planejamento e Gestão Estratégica, são utilizadas ferramentas quantitativas e qualitativas tanto para gerenciar os riscos do projeto, quanto para as ferramentas prospectivas. Para os gestores dos institutos de inovação e tecnologia, o foco das ferramentas é predominantemente qualitativo. Já o diretor corporativo afirmou que apenas técnicas qualitativas são utilizadas para apoiar a gestão de riscos nos projetos e ferramentas prospectivas.

\section{CONSIDERAÇÕES FINAIS}

A análise dos casos embasada na pesquisa teórica, análise documental e entrevistas aos gestores dos projetos dos institutos permitiu concluir que apesar de a metodologia documentada ser abrangente e estar alinhada aos preceitos dos referenciais teóricos abordadas nesta pesquisa, durante as entrevistas observou-se que o processo de gestão de riscos dos projetos estratégicos, de forma estruturada e sistêmica, ainda está em início de uso pela Fiesc.

Em relação ao objetivo de "analisar a possibilidade da aplicação conjunta da gestão de riscos em projetos estratégicos associado a ferramentas prospectivas, nos institutos de inovação e tecnologia do Sistema Fiesc", concluiu-se que é possível utilizá-las de forma integrada no planejamento, inclusive com ganhos consideráveis em termos de sinergia entre as ferramentas, ou seja, tanto as ferramentas prospectivas podem melhorar o processo de gestão de riscos em projetos, quanto os processos de gestão de riscos podem melhorar a utilização de ferramentas prospectivas, corroborando Koivisto et al. (2009). Em relação aos entrevistados, houve 
unanimidade quanto à possibilidade de aplicação conjunta da gestão de riscos em projetos e das ferramentas prospectivas, o que reforça a proposta deste estudo.

Quanto ao objetivo principal desta pesquisa - "analisar como é realizada a gestão de riscos e a utilização de ferramentas prospectivas no gerenciamento de projetos estratégicos nos institutos de inovação e tecnologia do Sistema Fiesc" -, a análise demonstrou que utilizar ferramentas prospectivas para gerenciar os riscos dos projetos dos institutos de inovação e tecnologia da Fiesc pode, além de melhorar o processo de identificação de riscos aos quais os projetos estão sujeitos, melhorar a proatividade do tratamento dos riscos, traçando planos de gestão de riscos e definindo roadmapping e planejamentos aos moldes do Masterplan.

Para concluir a pesquisa, fazem-se algumas recomendações relativas às observações e descobertas encontradas durante a pesquisa, em especial durante a análise do cruzamento das diversas fontes de informações relacionadas aos processos de gestão de riscos em projetos estratégicos do Sistema Fiesc, conforme segue:

- reforçar e tornar sistemáticos os processos de controle e monitoramento de riscos dos projetos estratégicos, em especial na fase posterior à identificação inicial de riscos, na qual foram encontradas as maiores lacunas em relação ao prescrito na documentação da organização;

- estabelecer processo formal de utilização das ferramentas prospectivas construídas, em especial o PDIC e os cenários integrados educação profissional e educação básica, inovação, serviços técnicos e tecnológicos, segurança e saúde no trabalho (2013/2027). Para este segundo estudo, também se recomenda que seja amplamente divulgado para os gestores da Fiesc e dos Projetos Estratégicos, em especial aos relacionados aos institutos de inovação e tecnologia da Fiesc, pois o estudo é muito alinhado às estratégias de negócio do Sistema Fiesc;

- melhorar o processo de análise quantitativa de risco em virtude do grande volume de investimentos que está sendo realizado nos Projetos dos Institutos de Inovação e Tecnologia da Fiesc. 
Por último, sugere-se a realização de estudos similares, para outros tipos de projetos estratégicos além daqueles dos institutos de inovação e tecnologia, com o objetivo de melhorar a gestão desses projetos e confirmar as constatações obtidas neste trabalho.

\section{REFERÊNCIAS}

Amer, M., Daim, T. U., \& Jetter, A. (2013). A review of scenario planning. Future, n. 46, 23-40.

Associação Brasileira de Normas Técnicas - ABNT. (2009). Gestão de riscos. NBR ISO 31000: Princípio e diretrizes. Rio de Janeiro: ABNT.

Barros, M. O., Werner, C. M. L., \& Travassos, G. H. (2004). Supporting risks in software project management. Journal of Systems and Software, $70(1), 21-35$.

Börjeson, L., Höjer, M., Dreborg, K. H., Ekvall, T., \& Finnveden, G. (2006). Scenario types and techniques: towards a user's guide. Futures, 38(7), 723-739.

Bradfield, R.; Wright, G., Burt, G., Cairns, G., \& Van der Heijden, K. (2005).The origins and evolution of scenario techniques in long range business planning. Futures, 37(8), 795-812.

Creswell, J. W. (1994). Research design: qualitative and quantitative approches. Thousend Oaks: Sage.

Dreborg, K. H. (2004). Scenarios and structural uncertainty. Stocholm: Department of Infrastructure. Royal Institute of Technology.

Ferreira, B. A. A., Almeida, J. O. R., Leão, P. R. C., \& Silva, N. P. G. (2013, setembro/dezembro). Gestão de riscos em projetos: uma análise comparativa da norma ISO 31000 e o Guia PMBOK®, 2012. Revista de Gestão e Projetos - GeP, 4(3), 46-72.

Federação das Indústrias de Santa Catarina - Fiesc. (2014). Programa de Desenvolvimento Industrial Catarinense: Plano estratégico 2015-2022: caderno executivo. Florianópolis: Fiesc. Recuperado em 18 de dezembro, 2014, de http://www4.fiescnet.com.br/images/banner-pedic/pdiccaderno-executivo.pdf

Gil, A. C. (1991). Métodos e técnicas de pesquisa social. São Paulo: Atlas. 
Gil, A. C. (1999). Métodos e técnicas de pesquisa social (5a ed.). São Paulo: Atlas.

Gomes, P. C. R. (2008). Amazônia dos rios: modelagem participativa da gestão do uso do solo para o empoderamento local. Tese de Doutorado, Centro de Desenvolvimento Sustentável, Universidade de Brasília, DF, Brasil.

Jouvenel, H. (2000, September). A brief methodological guide to scenario building. Technological Forecasting and Social Change, 65(1), 37-48.

Koivisto, R., Wessberg, N., Eerola, A., Ahlqvist, T., Kivisaari, S., Millyoja, J., \& Halonen, M. (2009). Integrating future-oriented technology analysis and risk assessment methodologies. Technological Forecasting and Social Change, 76(9), 1163-1176.

Kosko, B. (1986). Fuzzy cognitive maps. International Journal of ManMachine Studies, 24(1), 65-75.

Lopez, C., \& Salmeron, J. L. (2014). Dynamic risks modelling in ERP maintenance projects with FCM. Information Sciences, 256, 25-45.

López, C. (2012). Risks response strategies for supporting practitioners decision-making in software projects. Procedia Technology, 5, 437-444.

May, T. (2004). Pesquisa social: questões, métodos e processos. Porto Alegre: Artmed.

Motawa, I. A., Anumba, C. J., \& El-Hamalawi, A. (2006). A fuzzy system for evaluating the risk of change in construction projects. Advances in Engineering Software, 37(9), 583-591.

Palomo, J., Rios Insua, D., \& Ruggeri, F. (2007). Modeling external risks in project management. Risk Analysis, 27(4), 961-978.

Perminova, O., Gustafsson, M., \& Wikström, K. (2008). Defining uncertainty in projects- a new perspective. International Journal of Project Management, 26(1), 73-79.

Popper, R., Keenan, M., Miles, J., Butter, M., \& Sainz de la Fuenta, G. (2007). Global foresight outlook GFO 2007. Mapping Foresight in Europe and the rest of the World.

Popper, R. (2008). How are foresight methods selected? Foresight, 10(6), 62-89.

Project Management Institute - PMI. (2012). A guide to Project Management Body of knowledge - PMBOK (5th ed.). USA: PMI. 
Raz, T, Shenhar, A. J., \& Dvir, D. (2002). Risk management, project success, and technological uncertainty. $R \& D$ Management, 32(2), 101109.

Saritas, O. (2006). Systems thinking for foresight. Doctoral Thesis, The University of Manchester, UK.

Saritas, O., \& Aylen, J. (2010). Using scenarios for roadmapping: the case of clean production. Technological Forecasting \& Social Change, 77(7), 10611075.

Saritas, O., \& Miles, I. (2012). Scan-4-Light: a searchlight function horizon scanning and trend monitoring project. Foresight, 14(6), 489-510.

Saritas, O., \& Oner, M. A. (2004). Systemic analysis of UK foresight results: joint application of integrated management model and roadmapping. Technological Forecasting and Social Change, 71(1), 27-65.

Schoemaker, P. J.H. (1995, Winter). Scenario planning: a tool for strategic thinking. Sloan Management Review, 36(2), 25-40.

Schoemaker, P. J.H. (1991). When and how to use scenario planning: a heuristic approach with illustration. Journal of Forecasting, 10(6), 549564.

Serviço Nacional de Aprendizagem Industrial - Senai. (2013). Cenários integrados educação profissional e educação básica inovação e serviços técnicos e tecnológicos segurança e saúde no trabalho (2013/2027). Brasília: Senai/DN.

Van der Heijden, K. (1996). Scenarios: the art of strategic conversation. New York: John Wiley \& Sons.

Van Vliet, M., Kok, K., \& Veldkamp, T. (2010). Linking stakeholders and modellers in scenario studies: the use of Fuzzy Cognitive Maps as a communication and learning tool. Futures, 42(1), 1-14.

Vecchiato, R., \& Roveda, C. (2010). Strategic foresight in corporate organizations: handling the effect and response uncertainty of technology and social drivers of change. Technological Forecasting and Social Change, 77(9), 1527-1539.

Ward, S., \& Chapman, C. (2003). Transforming project risk management into project uncertainty management. International Journal of Project Management, 21(2), 97-105.

Wilkinson, A. (2009). Scenarios practices: in search of theory. Journal of Futures Studies, 13(3), 107-114. 
Yin, R. K. (2001). Estudo de caso: planejamento e métodos (2a ed.). Porto Alegre: Bookman.

Zeng, J., An, M., \& Smith, N. J. (2007). Application of a fuzzy based decision making methodology to construction project risk assessment.

International Journal of Project Management, 25(6), 589-600.

\section{APÊNDICE: ROTEIRO BÁSICO DE ENTREVISTA SEMIESTRUTURADA}

\begin{tabular}{|c|c|c|}
\hline CARACTERÍSTICAS & QUESTÕES & $\begin{array}{l}\text { REFERENCIAL } \\
\text { TEORICO/DOCUMENTAL }\end{array}$ \\
\hline \multirow{3}{*}{$\begin{array}{l}4.1 \text { - Analisar a } \\
\text { possibilidade da } \\
\text { aplicação conjunta } \\
\text { da Gestão de Riscos } \\
\text { em projetos } \\
\text { estratégicos } \\
\text { associada a } \\
\text { Ferramentas } \\
\text { Prospectivas, nos } \\
\text { Institutos de } \\
\text { Inovação e } \\
\text { Tecnologia do } \\
\text { Sistema Fiesc. }\end{array}$} & $\begin{array}{l}1 \text { - Como você percebe a } \\
\text { possibilidade de utilização integrada } \\
\text { das metodologias de Gestão de } \\
\text { Riscos e Ferramentas Prospectivas? } \\
2 \text { - Você percebe a utilização } \\
\text { integrada das metodologias de } \\
\text { Gestão de Riscos e Ferramentas } \\
\text { Prospectivas pelo Sistema Fiesc? } \\
3 \text { - Em sua opinião, isso auxilia na } \\
\text { Gestão de Riscos e, consequente } \\
\text { mente, na gestão do projeto? Se } \\
\text { sim, como isso pode ser } \\
\text { observado? }\end{array}$ & $\begin{array}{l}\text { 1) Ward e Chapman (2003); } \\
\text { 2) Barros et al. (2004); } \\
\text { 3) Saritas (2006); } \\
\text { 4) Motawa et al. (2006); } \\
\text { 5) Palomo, Rios Insua e Ruggeri } \\
\text { (2007); } \\
\text { 6) Perminova et al. (2008); } \\
\text { 7) Koivisto et al. (2009); } \\
\text { 8) Lopez e Salmeron (2014). }\end{array}$ \\
\hline & $\begin{array}{l}4 \text { - Como você avalia a resposta da } \\
\text { sociedade ao convite para que seus } \\
\text { representantes possam participar } \\
\text { desses estudos prospectivos } \\
\text { promovidos pelo Sistema Fiesc? }\end{array}$ & $\begin{array}{l}\text { 1) Schoemaker (1991; 1995); } \\
\text { 2) Van Der Heijden (1996); } \\
\text { 3) Bradfield, Wright, Burt, Cairns } \\
\text { e Van der Heijden (2005); } \\
\text { 4) Börjeson, Höjer, Dreborg, } \\
\text { Ekvall e Finnveden (2006); } \\
\text { 5) Popper, Keenan, Miles, Butter e } \\
\text { Sainz de la Fuenta } \\
\text { (2008); } \\
\text { 6) Koivisto et al. (2009); } \\
\text { 7) Wilkinson, (2009); } \\
\text { 8) Saritas e Aylen, (2010). }\end{array}$ \\
\hline & $\begin{array}{l}5 \text { - Você concorda que a fase da } \\
\text { implementação das Ferramentas } \\
\text { Prospectivas é a parte mais } \\
\text { complexa da gestão dos riscos em } \\
\text { projetos? Se sim, como o Sistema } \\
\text { Fiesc monitora a implantação de } \\
\text { estudos de cenários para mitigar os } \\
\text { riscos encontrados na fase de } \\
\text { concepção e ao longo da execução } \\
\text { do projeto? }\end{array}$ & $\begin{array}{l}\text { 1) Saritas e Oner, (2004); } \\
\text { 2) Saritas e Miles (2012). }\end{array}$ \\
\hline & $\begin{array}{l}6 \text { - Existe alguma ferramenta que } \\
\text { permita projeções quantitativas dos } \\
\text { riscos relacionados aos projetos? }\end{array}$ & $\begin{array}{l}\text { 1) Dreborg (2004); } \\
\text { 2) Börjeson et al., 2006); } \\
\text { 3) Gomes (2008); } \\
\text { 4) Van Vliet et al. (2010); } \\
\text { 5) Saritas e Miles (2012); } \\
\text { 6) Amer et al. (2013). } \\
\text { Análise Documental: } \\
\text { 1) Plano Estratégico Fiesc 2015- } \\
\text { 2022; } \\
\text { 2) Plano de Negócios; e } \\
\text { 3) Sistema Channel. }\end{array}$ \\
\hline
\end{tabular}




\begin{tabular}{|c|c|c|}
\hline CARACTERÍSTICAS & QUESTÕES & $\begin{array}{l}\text { REFERENCIAL } \\
\text { TEORICO/DOCUMENTAL }\end{array}$ \\
\hline \multirow[b]{2}{*}{$\begin{array}{l}4.2 \text { - Técnicas e } \\
\text { Ferramentas } \\
\text { Utilizadas }\end{array}$} & $\begin{array}{l}1 \text { - Quais as principais técnicas e } \\
\text { ferramentas são utilizadas para } \\
\text { gerencias os riscos? } \\
2 \text { - Quais as principais técnicas e } \\
\text { ferramentas são utilizadas para } \\
\text { elaborar e gerir os estudos } \\
\text { prospectivos utilizados? }\end{array}$ & $\begin{array}{l}\text { 1) Jouvenel (2000); } \\
\text { 2) Ward e Chapman (2003); } \\
\text { 3) Bradfield et al. (2005); } \\
\text { 4) Börjeson et al. (2006); } \\
\text { 5) Zeng et al. (2007); } \\
\text { 6) Popper et al. (2008); } \\
\text { 7) Ferreira et al. (2013); } \\
\text { 8) Koivisto et al. (2009). }\end{array}$ \\
\hline & $\begin{array}{l}2 \text { - A ferramenta Painel de } \\
\text { Especialistas foi utilizada em todos } \\
\text { os estudos prospectivo citados na } \\
\text { Análise da Documentação. Você } \\
\text { participou de algum Painel? Qual? }\end{array}$ & $\begin{array}{l}\text { Análise Documental: } \\
\text { 1) Programa de Desenvolvimento } \\
\text { da Indústria Catarinense (PDIC); } \\
\text { 2) Cenários integrados educação } \\
\text { profissional e educação básica } \\
\text { inovação e serviços técnicos e } \\
\text { tecnológicos segurança e saúde } \\
\text { no trabalho (2013/2027); } \\
\text { 3) Plano Estratégico Fiesc 2015- } \\
\text { 2022. }\end{array}$ \\
\hline \multirow[t]{2}{*}{$\begin{array}{l}4.3 \text { - Forma de } \\
\text { abordagem }\end{array}$} & $\begin{array}{l}1 \text { - Em relação à forma de abordar } \\
\text { os riscos e Ferramentas } \\
\text { Prospectivas, as técnicas e } \\
\text { ferramentas utilizadas são } \\
\text { preponderantemente Qualitativas } \\
\text { ou Quantitativas? }\end{array}$ & $\begin{array}{l}\text { 1) Jouvenel (2000); } \\
\text { 2) Ward e Chapman (2003); } \\
\text { 3) Dreborg (2004); } \\
\text { 4) Bradfield et al. (2005); } \\
\text { 5) Börjeson et al. (2006); } \\
\text { 6) Zeng et al. (2007); } \\
\text { 7) Popper et al. (2008); } \\
\text { 8) Koivisto et al. (2009); } \\
\text { 9) Vecchiato e Roveda (2010); } \\
\text { 10) Saritas e Miles (2012); } \\
\text { 11) Ferreira et al., (2013). }\end{array}$ \\
\hline & $\begin{array}{l}2 \text { - Como são realizadas as análises } \\
\text { quantitativas dos riscos dos } \\
\text { projetos? }\end{array}$ & $\begin{array}{l}\text { Análise Documental: } \\
\text { 1) Programa de Desenvolvimento } \\
\text { da Indústria Catarinense (PDIC); } \\
\text { 2) Cenários integrados educação } \\
\text { profissional e educação básica } \\
\text { inovação e serviços técnicos e } \\
\text { tecnológicos segurança e saúde } \\
\text { no trabalho (2013/2027); } \\
\text { 3) Plano Estratégico Fiesc 2015- } \\
\text { 2022. }\end{array}$ \\
\hline
\end{tabular}

